\title{
ENVIRONMENTAL TEST ACTIVITY ON THE FLIGHT MODULES OF THE GLAST LAT TRACKER
}

\author{
M.BRIGIDA, A.CALIANDRO, C.FAVUZZI, P.FUSCO, F.GARGANO, \\ N.GIGLIETTO, F.GIORDANO, F.LOPARCO, B.MARANGELLI, M.N.MAZZIOTTA, \\ N. MIRIZZI, S.RAINÖ* \\ For the GLAST Collaboration \\ Dipartimento Interateneo di Fisica, Università di Bari and INFN-Bari, \\ Via Orabona, 4 \\ Bari, Italy
}

\begin{abstract}
The GLAST Large Area Telescope (LAT) is a gamma-ray telescope consisting of a silicon micro-strip detector tracker followed by a segmented CsI calorimeter and covered by a segmented scintillator anticoincidence system that will search for $\gamma$-rays in the 20 MeV-300 GeV energy range. The results of the environmental tests performed on the flight modules (towers) of the Tracker are presented. The aim of the environmental tests is to verify the performance of the silicon detectors in the expected mission environment. The tower modules are subjected to dynamic tests that simulate the launch environment and thermal vacuum test that reproduce the thermal gradients expected on orbit. The tower performance is continuously monitored during the whole test sequence. The environmental test activity, the results of the tests and the silicon tracker performance are presented.
\end{abstract}

\section{Introduction}

GLAST, the Gamma Ray Large Area Space Telescope, is a high energy gammaray astronomy mission planned for launch in September 2007. It is composed of two main instruments: the Large Area Telescope (LAT), to search for $\gamma$-rays in the energy range from $20 \mathrm{MeV}$ to $300 \mathrm{GeV}$, and the Gamma-ray Burst Monitor (GBM) for high variability phenomena studies at lower energies. The LAT is a gamma-ray telescope consisting of a silicon micro-strip detector tracker followed by a segmented CsI calorimeter, to reconstruct $\gamma$-rays direction and energy. The

tracker and the calorimeter are covered by a segmented scintillator anticoincidence system to reject charged particle background [1]. 
The Large Area Telescope is based on the conversion of gamma-rays into electron-positron pairs and is arranged in a $4 \times 4$ modular array of towers, consisting of one Tracker and one Calorimeter module. Each Tracker module (that will be referred to as a "tower", from now on) corresponds to a stack of 19 carbon fiber panels (trays) supporting the silicon strip detectors and the electronics. With the exceptions of the top and bottom trays of the tower, each tray supports silicon detectors both on upper and lower faces. A more detailed description of the LAT instruments can be found at reference [1].

\section{Environmental Test flow of the LAT Tracker Towers}

All the trays and all the towers have been subjected to an environmental tests sequence before being delivered to SLAC for the Tracker assembly to verify that all of them are acceptable for flight and can operate in space environment. At the moment these proceedings are written (October 2005), all the 16 flight towers have been assembled, tested and delivered to SLAC, where 14 of them have already been assembled in the flight grid structure.

After each tower assembly, the environmental test sequence consists in the performance of vibration and thermal vacuum tests preceded and followed by functional tests to verify the electrical integrity and optimum functionality of silicon detectors and electronics after dynamical loads and thermal transitions. These tests have been performed in the Alenia-Alcatel Assembly, Integration and Test facility in Rome. The GLAST INFN-Bari group is responsible for the environmental testing of the TKR towers, completed in October 2005.

\subsection{Vibration Test}

The goal of the vibration tests is to demonstrate that the hardware is acceptable for flight and that it will survive to the environments imposed during the launch. This is accomplished by subjecting the flight Tracker modules to tests at acceptance levels. LDS shake tables are used for testing the towers.

The transfer function of the towers is evaluated by subjecting it to low level signature sweep test from 5 to $2000 \mathrm{~Hz}$ that are performed before and after each dynamic proof to compare the tower behaviour when it is subjected to the dynamic environment. Changes in the fundamental frequency and/or amplitude are used to identify possible structural damage that may have occurred during the dynamic test sequence. Figure 1 shows the responses of one flight TKR tower to the pre- and post-test low level signature sweeps along the $\mathrm{Z}$ axis: the first normal mode is at about $370 \mathrm{~Hz}$. 
The dynamic environments are simulated by sinusoidal vibration in the 5 to $50 \mathrm{~Hz}$ frequency range and random vibration from 20 to $2000 \mathrm{~Hz}$. The response under dynamic excitation is studied both along the thrust axis (Z) first and lateral directions $(\mathrm{X}, \mathrm{Y})$ successively.

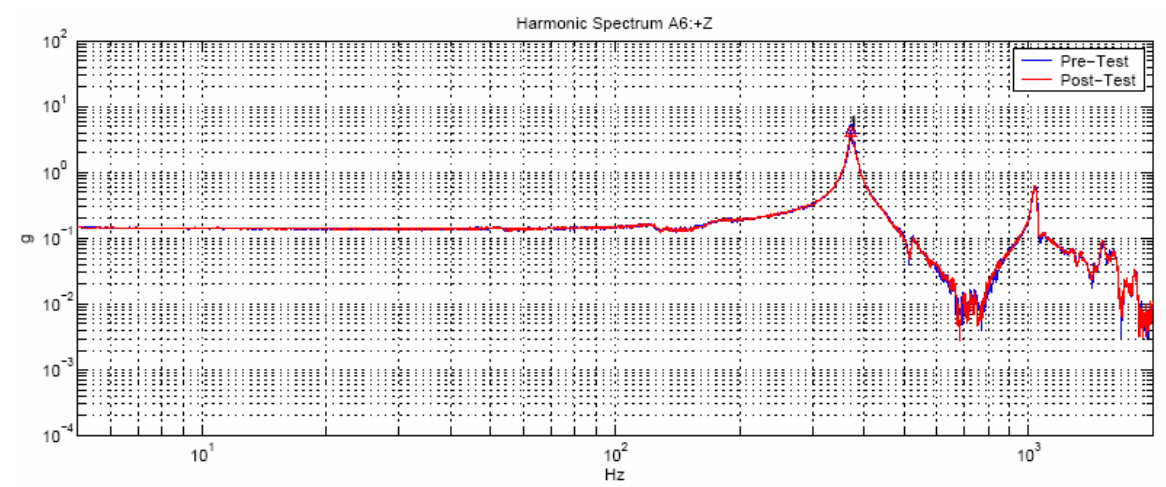

Figure 1 Pre-test (blue line) and post test (red line) responses to low level signature sweep.

The random vibration is performed at various levels starting from $-12 \mathrm{~dB}$, $6 \mathrm{~dB}$ up to the full level with an input spectrum of $6.8 \mathrm{~g}$ rms. A summary plot showing the $\mathrm{Z}$ axis normal modes and $\mathrm{Q}$ values of all the flight towers tested is shown in Figure 2. All the normal mode frequencies measured are between 350 and $400 \mathrm{~Hz}$ [2].

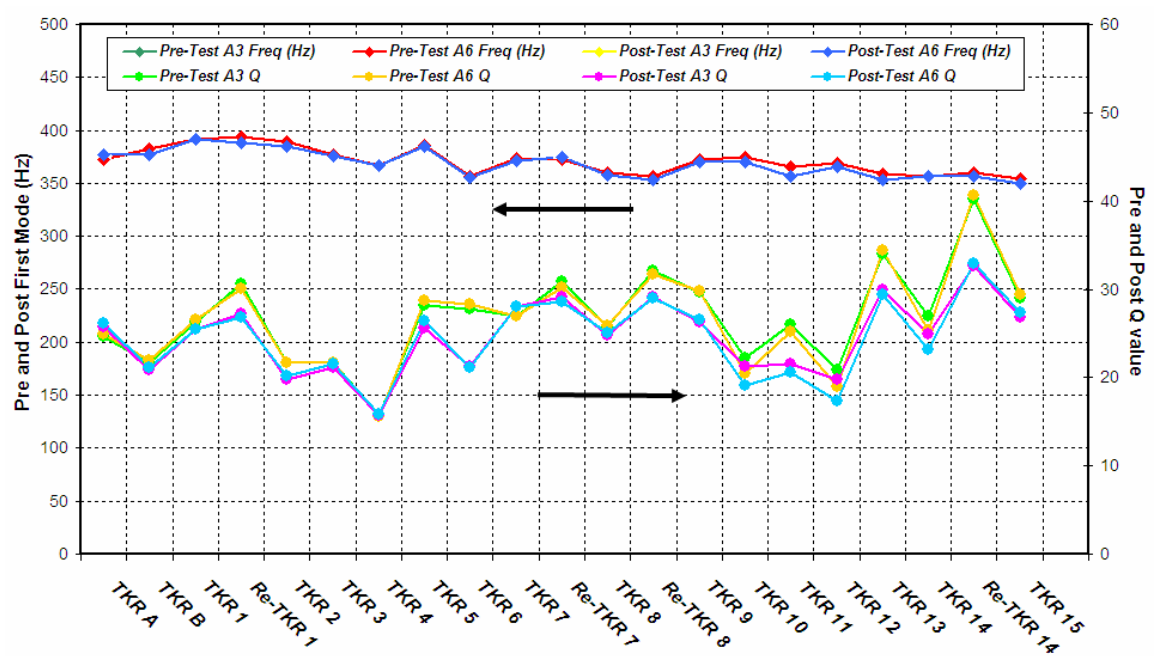

Figure 2 Summary plot of the first mode frequencies and Q values for all the flight TKR towers. 


\subsection{Thermal vacuum tests}

Thermal vacuum tests on flight hardware are performed to demonstrate that the tower modules will get over the thermal gradients expected during the mission and that the functional capability of the hardware is not degraded by thermal transients. For each tower four thermal cycles are performed in the $-15^{\circ} \mathrm{C} \div$ $+45^{\circ} \mathrm{C}$ temperature range at a vacuum level of $10^{-5}$ Torr. A cold plate is used to drive the towers in temperature by conduction. Moreover, heaters located on an external box surrounding the towers provided a better warming up of the modules.

Functional tests are performed during transients and at hot/cold plateaus to check the silicon detectors performance as a function of temperature. Moreover, at each temperature extremes the Turn Off/Turn On capability is verified. The temperature is monitored by means of thermocouples located on the tower sidewalls and on the ground support equipment and, also, by means of the thermistors located on the tower flex cables. Figure 3 shows the temperature profile as a function of time measured by the thermistors [2].

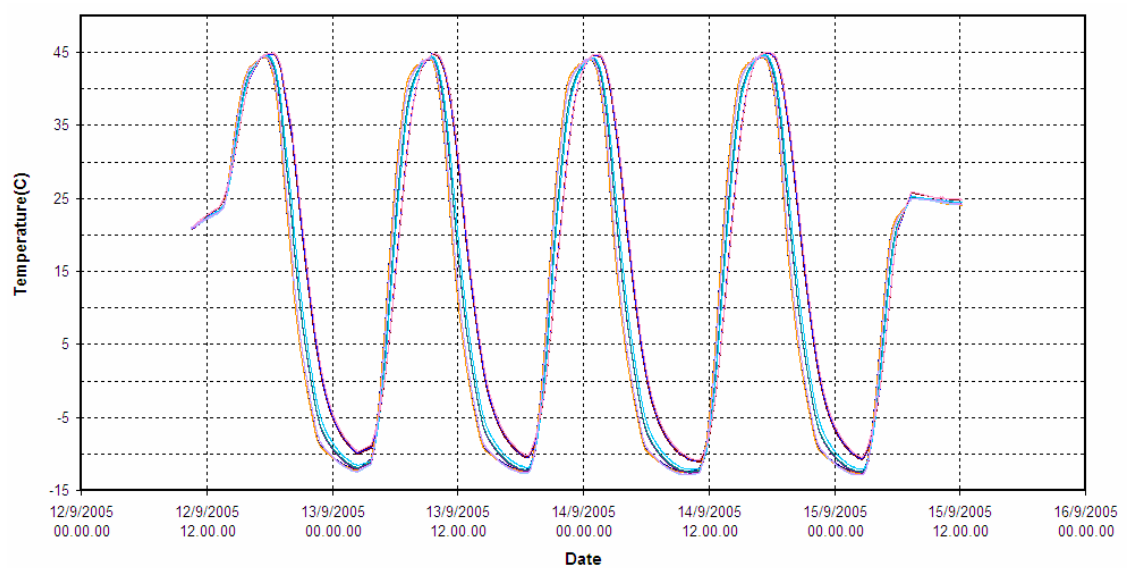

Figure 3 Temperature profile measured by the tower thermistors as a function of time.

\subsection{Functional tests}

Electrical tests are performed before and after all the environmental proofs. Comprehensive Performance Tests (CPT) and Limited Performance Tests (LPT) include the verification of the load and read-back capability of registers and front-end ASICs, the complete monitoring of the noise, gain and noise occupancy of all the channels in a Tracker module $(\sim 55000)$, the check of trigger lines, cosmic rays data acquisition. 
The main objective of the functional tests is to verify that the tower performance is in conformance with the requirements established for the detector. The measurement of the gain and noise of all the channels of a tower allows to identify the dead strips (gain less than $50 \mathrm{mV} / \mathrm{fC}$ )) and the disconnected strips (noise less than 500 ENC). The single strips noise occupancy test searches for the noisy strips: any channel with a noise occupancy larger than $10^{-3}$ is considered noisy.

Functional tests are also continuously performed during thermal vacuum transients and at hot/cold dwell points $\left(-15^{\circ} \mathrm{C},+45^{\circ} \mathrm{C}\right)$ in order to study how the SSDs parameters change with temperature. The summary plot of the disconnected channels measured before, during and after one tower test is shown in Figure 4 .

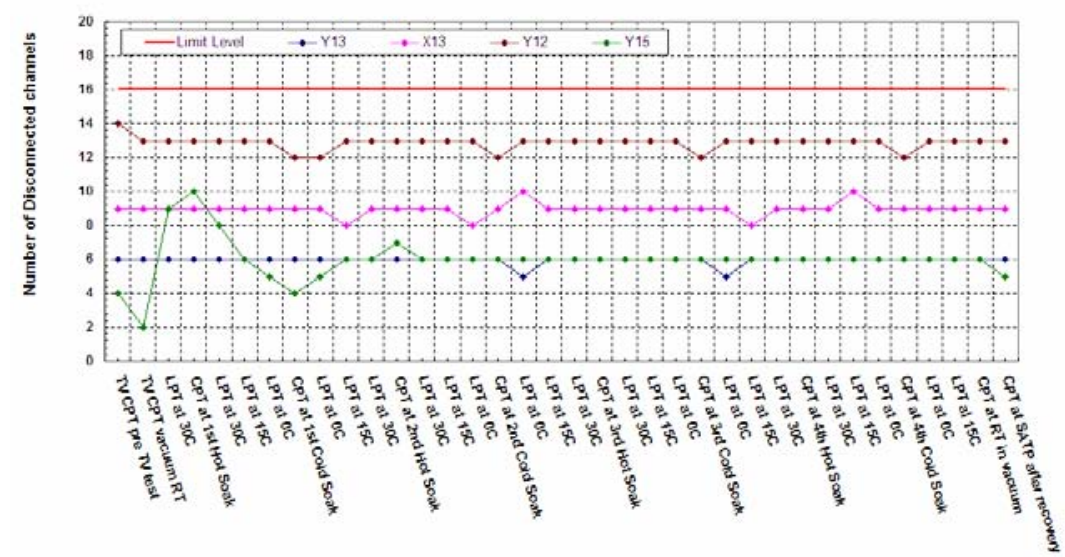

Figure 4 Summary of the disconnected channels identified in a complete sequence of functional tests before during and after a thermal vacuum test.

\section{Conclusions}

The environmental test sequence including dynamic loads, thermal vacuum cycles and functional tests have been successfully performed on all the 16 flight Tracker modules. The assembly of the LAT Tracker will be completed at SLAC by the end of October 2005. GLAST launch is scheduled for September 2005.

\section{References}

1. http://www-glast.slac.stanford.edu/pubfiles/proposals/bigprop

2. http://www.ba.infn.it/ glast/env test.htm GLAST Bari Environmental Test Web Page

3. http://www-glast.slac.stanford.edu/ GLAST LAT Internal Document: LATTD-00191-02 